Скопје, Македонија

\title{
GENERALIZED POTENTIAL INEQUALITY AND EXPONENTIAL CONVEXITY
}

\author{
NEVEN ELEZOVIĆ, JOSIP PEČARIĆ, AND MARJAN PRALJAK*
}

\begin{abstract}
In this paper we generalize the potential inequality which was introduced in [6] and extended to the class of naturally defined convex functions in [1]. The generalization is achieved by replacing the 1st order Taylor expansion of a convex function in the proof of the potential inequality with the $n$-th order Taylor expansion of an $(n+1)$-convex function.

Furthermore, by using methods developed in [4] and [2] we construct several families of $n$-exponentially convex functions by making use of linearity of the generalized potential inequality.
\end{abstract}

\section{INTRODUCTION}

Potential inequality, introduced by Rao and Šikić in [6], is a very general inequality that holds for kernels that satisfy the maximum principle (see next section), an important property from the potential theory that is satisfied by many classical kernels. Rao and Šikić proved the inequality only for a special class of convex and concave functions, which was later extended to the naturally defined class of convex (and concave) functions by Elezović, Pečarić and Praljak in [1]. The latter form of the potential inequality is given in the following theorem (Theorem 5 in [1])

Theorem 1 (The potential inequality for convex functions). Let $g:(0,+\infty)$ $\rightarrow \mathbb{R}$ be a convex function, $g^{\prime}$ its right-continuous derivative and $N(x, d y)$ a positive kernel on $X$ which satisfies the strong maximum principle on $\mathcal{R}$ with constant $M$. Let $f \in \mathcal{R}, x \in X$ and $z>0$ be such that $z \leq(N f)(x) / M$

2010 Mathematics Subject Classification. 26D15.

Key words and phrases. Potential inequality, n-convex function, exponential convexity.

${ }^{*}$ Corresponding author 
and denote by $B_{z}$ the set

$$
B_{z}=\{y \in X:(N f)(y) \geq z\} .
$$

Then

$$
\begin{aligned}
g\left(\frac{1}{M}(N f)(x)\right)-g(z) \leq & \frac{1}{M} N\left[f^{+} g^{\prime}(N f) \mathbf{1}_{B_{z}}\right](x) \\
& +\frac{1}{M} g^{\prime}(z) N\left[f-f^{+} \mathbf{1}_{B_{z}}\right](x)-z g^{\prime}(z) .
\end{aligned}
$$

In addition to the maximum principle, another crucial step in the proof of the potential inequality is an integration by parts which gives the 1st order Taylor expansion of the convex function with the remainder in its integral form. In this paper we will generalize the potential inequality by replacing the 1st order Taylor expansion of the convex function with the $n$-th order Taylor expansion of an $(n+1)$-convex function. Furthermore, the differences generated by the generalized potential inequality are linear functionals and, by using methods developed in [3], [2] and [4], we construct several families of exponentially convex functions.

\section{Generalized potential inequality}

We will first introduce the notation and the set up. We say that $N(x, d y)$ is a (positive) kernel on $X$ if $N: X \times \mathcal{B}(X) \rightarrow[0,+\infty]$ is a mapping such that, for every $x \in X, A \mapsto N(x, A)$ is a $\sigma$-finite measure, and, for every $A \in \mathcal{B}(X), x \mapsto N(x, A)$ is a measurable function. For a measurable function $f$, the potential of $f$ with respect to $N$ at a point $x \in X$ is

$$
(N f)(x)=\int_{X} f(y) N(x, d y),
$$

whenever the integral exists. The class of functions that have the potential at every point is denoted by $\mathcal{P O} \mathcal{T}(N)$.

For a measure $\mu$ on $(X, \mathcal{B}(X))$, a measurable set $C \in \mathcal{B}(X)$ and $k \in \mathbb{N}_{0}$ we will denote by $\hat{N}_{C}^{k} \mu$ the measure defined by

$$
\left(\hat{N}_{C}^{k} \mu\right)(d y)=\int_{C} N(x, X)^{k} N(x, d y) \mu(d x) .
$$

If $C=X$ we will omit the subscript, i. e. $\hat{N}^{k} \mu$ will denote the measure $\hat{N}_{X}^{k} \mu$.

Definition 1. Let $N$ be a positive kernel on $X$ and $\mathcal{R} \subset \mathcal{P O} \mathcal{T}(N)$. We say that $N$ satisfies the strong maximum principle on $\mathcal{R}$ (with constant 
$M \geq 1)$ if

$$
(N f)(x) \leq M u+N\left[f^{+} \mathbf{1}_{\{(N f) \geq u\}}\right](x)
$$

holds for every $x \in X, f \in \mathcal{R}$ and $u \geq 0$.

We will also make use of the divided differences. Let $g$ be a real-valued function on an interval $I \subset \mathbb{R}$. The divided difference of order $n$ of the function $g$ at distinct points $z_{0}, z_{1}, \ldots, z_{n} \in I$ is defined (see [5]) recursively by

$$
\left[z_{i}\right] g=g\left(z_{i}\right), \quad i=0, \ldots, n
$$

and

$$
\left[z_{0}, z_{1}, \ldots, z_{n}\right] g=\frac{\left[z_{1}, \ldots, z_{n}\right] g-\left[z_{0}, \ldots, z_{n-1}\right] g}{z_{n}-z_{0}} .
$$

The value $\left[z_{0}, \ldots, z_{n}\right] g$ is independent of the order of the points $z_{0}, \ldots, z_{n}$. The definition may be extended to include the case in which some (or all) of the points coincide by assuming $z_{0} \leq z_{1} \leq \ldots \leq z_{n}$ and letting

$$
[\underbrace{z, \ldots, z}_{j \text {-times }}] g=\frac{g^{(j-1)}(z)}{(j-1) !}
$$

provided that $f^{(j-1)}(z)$ exists.

A function $g$ is said to be $n$-convex, $n \geq 0$, if the $n$-th order divided difference satisfies

$$
\left[z_{0}, \ldots, z_{n}\right] g \geq 0, \quad \text { for all choices of distinct points } z_{0}, \ldots, z_{n} \in I \text {. }
$$

If a function $g$ is $(n+1)$-convex, $n \geq 1$, then the derivatives $g^{(k)}$ exist for $1 \leq k \leq n-1$, while for $k=n$ the right sided derivative $g_{+}^{(n)}$ exists and it is right-continuous and non-decreasing (see [5], Theorem 1.41). We will denote this right sided derivative simply by $g^{(n)}$ and $d g^{(n)}$ will denote the positive measure generated by $g^{(n)}$.

Theorem 2 (Generalized potential inequality). Let $g:(0,+\infty) \rightarrow \mathbb{R}$ be an $(n+1)$-convex function and $N(x, d y)$ a positive kernel on $X$ which satisfies the strong maximum principle on $\mathcal{R}$ with constant $M$. Let $f \in \mathcal{R}, x \in X$ and $z>0$ be such that $z \leq(N f)(x) / M<+\infty$ and let the set $B_{z}$ be defined 
by (1). Then

$$
\begin{aligned}
& g\left(\frac{1}{M}(N f)(x)\right)-g(z) \leq \frac{N(x, X)^{n-1}}{n ! M^{n}}\left\{N\left[\left(f^{+}\right)^{n} g^{(n)}(N f) \mathbf{1}_{B_{z}}\right](x)\right. \\
& \left.-g^{(n)}(z) N\left[\left(f^{+}\right)^{n} \mathbf{1}_{B_{z}}\right](x)\right\}+\sum_{i=1}^{n} \frac{1}{i !}\left(\frac{1}{M}(N f)(x)-z\right)^{i} g^{(i)}(z)
\end{aligned}
$$

Proof. The $n$-th order Taylor expansion of the function $g$ is

$$
g(\tau)-g(z)=\sum_{i=1}^{n} \frac{1}{i !}(\tau-z)^{i} g^{(i)}(z)+R_{n}(z),
$$

where the remainder $R_{n}$ in its integral form is given by

$$
R_{n}(z)=\frac{1}{n !} \int_{z}^{\tau}(\tau-u)^{n} d g^{(n)}(u)
$$

Inserting $\tau=\tau(x)=\frac{1}{M}(N f)(x)$ in (3) and using the strong maximum principle under the integral of the remainder, since $d g^{(n)}(u)$ is a positive measure, we get

$$
\begin{aligned}
& g(\tau(x))-g(z) \leq \\
& \quad \leq \frac{1}{n ! M^{n}} \int_{z}^{\tau(x)} N\left[f^{+} \mathbf{1}_{\{N f \geq u\}}\right]^{n} d g^{(n)}(u)+\sum_{i=1}^{n} \frac{1}{i !}(\tau(x)-z)^{i} g^{(i)}(z) .
\end{aligned}
$$

We can bound the integral on the right hand side of the last inequality by applying, respectively, Jensen's inequality, Fubini's theorem and the fact that $\left(f^{+}\right)^{n} \mathbf{1}_{\{N f \geq u\}}$ is a nonnegative function from the third to the fifth line below

$$
\begin{aligned}
\int_{z}^{\tau(x)} & N\left[f^{+} \mathbf{1}_{\{N f \geq u\}}\right]^{n} d g^{(n)}(u) \\
& =\int_{z}^{\tau(x)}\left[\int_{X} f^{+}(y) \mathbf{1}_{\{(N f) \geq u\}}(y) N(x, d y)\right]^{n} d g^{(n)}(u) \\
& \leq \int_{z}^{\tau(x)}\left(\int_{X} N(x, d y)\right)^{n-1}\left(\int_{X} f^{+}(y)^{n} \mathbf{1}_{\{N f \geq u\}}(y)^{n} N(x, d y)\right) d g^{(n)}(u)
\end{aligned}
$$




$$
\begin{aligned}
& =N(x, X)^{n-1} \int_{X}\left[\int_{z}^{\tau(x)} \mathbf{1}_{\{N f \geq u\}}(y) d g^{(n)}(u)\right] f^{+}(y)^{n} N(x, d y) \\
& \leq N(x, X)^{n-1} \int_{X}\left[\int_{z}^{+\infty} \mathbf{1}_{\{N f \geq u\}}(y) d g^{(n)}(u)\right] f^{+}(y)^{n} N(x, d y) \\
& =N(x, X)^{n-1} \int_{X} f^{+}(y)^{n}\left[g^{(n)}((N f)(y))-g^{(n)}(z)\right] \mathbf{1}_{B_{z}}(y) N(x, d y) \\
& =N(x, X)^{n-1}\left\{N\left[\left(f^{+}\right)^{n} g^{(n)}(N f) \mathbf{1}_{B_{z}}\right](x)-g^{(n)}(z) N\left[\left(f^{+}\right)^{n} \mathbf{1}_{B_{z}}\right](x)\right\} .
\end{aligned}
$$

Finally, combining the last inequality with (4) finishes the proof.

Let us further denote the set

$$
B=\bigcup_{z>0} B_{z}=\{x \in X:(N f)(x)>0\}
$$

The integral version of the generalized potential inequality is obtained by integrating the inequality from Theorem 2 with respect to the variable $x$.

Corollary 3. Let the assumptions of Theorem 2 hold for a function $z$ : $B \rightarrow(0,+\infty)$, i. e. $z(x) \leq(N f)(x) / M$ for $x \in B$. Then, for $C \subset B$, $C \in \mathcal{B}(X)$, and a finite measure $\mu$ on $(X, \mathcal{B}(X))$, the following inequality holds

$$
\begin{aligned}
& \int_{C}\left(g\left(\frac{1}{M}(N f)(x)\right)-g(z(x))\right) \mu(d x) \\
& \leq \frac{1}{n ! M^{n}} \int_{C}\left(\int_{B_{z(x)}} f^{+}(y)^{n} g^{(n)}((N f)(y)) N(x, d y)\right) N(x, X)^{n-1} \mu(d x) \\
& \quad-\frac{1}{n ! M^{n}} \int_{C}\left(\int_{B_{z(x)}} f^{+}(y)^{n} N(x, d y)\right) g^{(n)}(z(x)) N(x, X)^{n-1} \mu(d x) \\
& \quad+\sum_{i=1}^{n} \frac{1}{i !} \int_{C}\left(\frac{1}{M}(N f)(x)-z(x)\right)^{i} g^{(i)}(z(x)) \mu(d x) .
\end{aligned}
$$


In particular, for $C=B_{z}$ and the constant function $z(x) \equiv z$ we get

$$
\begin{gathered}
\int_{B_{z}} g\left(\frac{1}{M}(N f)(x)\right) \mu(d x)-g(z) \mu\left(B_{z}\right) \\
\leq \frac{1}{n ! M^{n}} \int_{B_{z}} f^{+}(x)^{n} g^{(n)}((N f)(x))\left(\hat{N}_{B_{z}}^{n-1} \mu\right)(d x) \\
-\frac{g^{(n)}(z)}{n ! M^{n}} \int_{B_{z}} f^{+}(x)^{n}\left(\hat{N}_{B_{z}}^{n-1} \mu\right)(d x)+\sum_{i=1}^{n} \frac{g^{(i)}(z)}{i !} \int_{B_{z}}\left(\frac{1}{M}(N f)(x)-z\right)^{i} \mu(d x) .
\end{gathered}
$$

Proof. Integrating the generalized potential inequality with respect to the measure $\mu$ we get

$$
\begin{gathered}
\int_{C}\left(g\left(\frac{1}{M}(N f)(x)\right)-g(z(x))\right) \mu(d x) \\
\leq \frac{1}{n ! M^{n}} \int_{C} N(x, X)^{n-1} N\left[\left(f^{+}\right)^{n} g^{(n)}(N f) \mathbf{1}_{B_{z(x)}}\right](x) \mu(d x) \\
\quad-\frac{1}{n ! M^{n}} \int_{C} N(x, X)^{n-1} g^{(n)}(z(x)) N\left[\left(f^{+}\right)^{n} \mathbf{1}_{B_{z(x)}}\right](x) \mu(d x) \\
\quad+\sum_{i=1}^{n} \frac{1}{i !} \int_{C}\left(\frac{1}{M}(N f)(x)-z(x)\right)^{i} g^{(i)}(z(x)) \mu(d x) .
\end{gathered}
$$

which is the first inequality.

The second inequality follows by taking $C=B_{z}$ and $z(x) \equiv z$ and by applying Fubini's theorem on the first two integrals of the right hand side.

Corollary 4. Under the assumptions of Corollary 3, for $p \in \mathbb{R} \backslash\{0,1, \ldots, n\}$ the following inequalities hold:

$$
\begin{aligned}
& \frac{1}{p(p-1) \cdots(p-n)} \int_{B_{z}}(N f)^{p}(x) \mu(d x) \leq \\
& \frac{M^{p-n}}{n !(p-n)} \int_{B_{z}} f^{+}(x)^{n}(N f)^{p-n}(x)\left(\hat{N}_{B_{z}}^{n-1} \mu\right)(d x) \\
& \quad-\frac{(z M)^{p-n}}{n !(p-n)} \int_{B_{z}} f^{+}(x)^{n}\left(\hat{N}_{B_{z}}^{n-1} \mu\right)(d x) \\
& +\sum_{i=0}^{n} \frac{z^{p-i} M^{p}}{i !(p-i)(p-i-1) \cdots(p-n)} \int_{B_{z}}\left(\frac{1}{M}(N f)(x)-z\right)^{i} \mu(d x)
\end{aligned}
$$


and

$$
\begin{aligned}
& \frac{1}{p(p-1) \cdots(p-n)} \int_{B_{z}}(N f)^{p}(x) \mu(d x) \leq \\
& \frac{M^{p-n}}{n !(p-n)}\left[\int_{B_{z}}\left(f^{+}\right)^{p} d\left(\hat{N}_{B_{z}}^{n-1} \mu\right)\right]^{\frac{n}{p}}\left[\int_{B_{z}}(N f)^{p} d\left(\hat{N}_{B_{z}}^{n-1} \mu\right)\right]^{1-\frac{n}{p}} \\
& \quad-\frac{(z M)^{p-n}}{n !(p-n)} \int_{B_{z}} f^{+}(x)^{n}\left(\hat{N}_{B_{z}}^{n-1} \mu\right)(d x) \\
& +\sum_{i=0}^{n} \frac{z^{p-i} M^{p}}{i !(p-i)(p-i-1) \cdots(p-n)} \int_{B_{z}}\left(\frac{1}{M}(N f)(x)-z\right)^{i} \mu(d x) .
\end{aligned}
$$

Proof. The first inequality is obtained by applying the second inequality from Corollary 3 for $(n+1)$-convex functions

$$
g_{p}(z)=\frac{1}{p(p-1) \cdots(p-n)} z^{p}
$$

for $p \in \mathbb{R} \backslash\{0,1, \ldots n\}$, and rearranging.

The second inequality follows from the first by applying Hölder's inequality on the first integral of the right hand side for the pair of conjugate exponents $p / n$ and $p /(p-n)$.

If Theorem 2 holds for $z>0$, then it holds for every $z^{\prime}, 0<z^{\prime} \leq z$. Letting $z^{\prime} \rightarrow 0$, if the function $g$ satisfies certain properties, we can get further inequalities.

In the following theorem we will assume that either $g^{(n)}$ is nonnegative, or that for every $x \in B$ there exists a function $h_{x} \in L^{1}(N(x, \cdot))$ such that $\left|\left(f^{+}\right)^{n} g^{(n)}(N f)\right| \leq h_{x}$. In either case, by the monotone convergence theorem in the former and by the dominated convergence theorem in the latter, we have

$$
\lim _{z \searrow 0} N\left[\left(f^{+}\right)^{n} g^{(n)}(N f) \mathbf{1}_{B_{z}}\right]=N\left[\left(f^{+}\right)^{n} g^{(n)}(N f) \mathbf{1}_{B}\right]
$$

since $\left(f^{+}\right)^{n} g^{(n)}(N f) \mathbf{1}_{B_{z}} \rightarrow\left(f^{+}\right)^{n} g^{(n)}(N f) \mathbf{1}_{B}$ pointwise, when $z \rightarrow 0$. 
Corollary 5. Under the assumptions of Theorem 2, if $g^{(i)}(0+)$ is finite for $i=0,1, \ldots, n$, then for every $x \in B$ we have

$$
\begin{aligned}
g\left(\frac{1}{M}(N f)(x)\right) & \leq \frac{N(x, X)^{n-1}}{n ! M^{n}}\left\{N\left[\left(f^{+}\right)^{n} g^{(n)}(N f) \mathbf{1}_{B}\right](x)\right. \\
& \left.-g^{(n)}(0+) N\left[\left(f^{+}\right)^{n} \mathbf{1}_{B}\right](x)\right\}+\sum_{i=0}^{n} \frac{1}{i ! M^{i}}(N f)(x)^{i} g^{(i)}(0+) .
\end{aligned}
$$

Furthermore, if $\mu$ is a finite measure on $(X, \mathcal{B}(X))$, then the following inequality holds

$$
\begin{gathered}
\int_{B} g\left(\frac{1}{M}(N f)(x)\right) \mu(d x) \leq \frac{1}{n ! M^{n}} \int_{B} f^{+}(x)^{n} g^{(n)}((N f)(x))\left(\hat{N}_{B}^{n-1} \mu\right)(d x) \\
-\frac{g^{(n)}(0+)}{n ! M^{n}} \int_{B} f^{+}(x)^{n}\left(\hat{N}_{B}^{n-1} \mu\right)(d x)+\sum_{i=0}^{n} \frac{g^{(i)}(0+)}{i ! M^{i}} \int_{B}(N f)(x)^{i} \mu(d x) .
\end{gathered}
$$

Proof. The first inequality follows from Theorem 2 by letting $z \rightarrow 0$ and rearranging. The second inequality follows by integrating the first with respect to the measure $\mu$ over the set $B$ and applying Fubini's theorem on the first two integrals of the righ hand side.

Corollary 6. Under the assumptions of Corollary 5, for $p>n$ the following inequalities hold:

$$
\begin{aligned}
& \int_{B}(N f)^{p}(x) \mu(d x) \leq \\
& \quad \frac{p(p-1) \cdots(p-n+1) M^{p-n}}{n !} \int_{B} f^{+}(x)^{n}(N f)^{p-n}(x)\left(\hat{N}_{B}^{n-1} \mu\right)(d x)
\end{aligned}
$$

and

$$
\begin{aligned}
& \int_{B}(N f)^{p}(x) \mu(d x) \leq \\
& \frac{p(p-1) \cdots(p-n+1) M^{p-n}}{n !}\left[\int_{B_{z}}\left(f^{+}\right)^{p} d\left(\hat{N}_{B_{z}}^{n-1} \mu\right)\right]^{\frac{n}{p}}\left[\int_{B}(N f)^{p} d\left(\hat{N}_{B}^{n-1} \mu\right)\right]^{1-\frac{n}{p}} .
\end{aligned}
$$

Proof. The first inequality holds since functions $g_{p}$ given by (5) for $p>n$ satisfy the assumptions of Corollary 5 with $g^{(i)}(0+)=0$ for $i=0,1, \ldots, n$. The second inequality follows from the first by applying Hölder's inequality on the right hand side integral with the pair of conjugate exponents $p / n$ and $p /(p-n)$. 
Example 7 (Application to a Hardy-type kernel). Let $X=(0,+\infty)$ and let the kernel $N(x, d y)=G(x, y) d y$ be given by its density

$$
G(x, y)= \begin{cases}1, & \text { if } y \leq x \\ 0, & \text { otherwise }\end{cases}
$$

The kernel $N$ satisfies the maximum principle with constant $M=1$ (see $[6])$,

$$
F(x)=(N f)(x)=\int_{0}^{x} f(y) d y
$$

and

$$
N(x, X)=\int_{0}^{+\infty} G(x, y) d y=\int_{0}^{x} d y=x .
$$

For a nonnegative function $f$ the set $B$ is equal to

$$
B=\{x \in X:(N f)(x)>0\}=\{x \in(0,+\infty): F(x)>0\}=(b,+\infty),
$$

where $b=\operatorname{ess} \inf \{y: f(y)>0\}$.

Further, let $\nu_{i}(d x)=\lambda_{i}(x) d x, i=1,2$, be two $\sigma$-finite measures with densities $\lambda_{i}$ that satisfy

$$
\lambda_{2}(x)=\lambda_{1}(x)^{1-\frac{p}{n}}\left[\int_{x}^{+\infty} y^{n-1} \lambda_{1}(y) d y\right]^{\frac{p}{n}} .
$$

The measure $\hat{N}_{B}^{n-1} \nu_{1}$ satisfies

$$
\begin{array}{r}
\left(\hat{N}_{B}^{n-1} \nu_{1}\right)(d x)=\int_{b}^{+\infty} N(y, X)^{n-1} N(y, d x) \lambda_{1}(y) d y= \\
=\int_{b}^{+\infty} y^{n-1}\left[\int_{0}^{y} d x\right] \lambda_{1}(y) d y=\int_{0}^{+\infty}\left[\int_{\max (b, x)}^{+\infty} y^{n-1} \lambda_{1}(y) d y\right] d x
\end{array}
$$

i. e.

$$
d\left(\hat{N}_{B}^{n-1} \nu_{1}\right)(x)=\int_{\max (b, x)}^{+\infty} y^{n-1} \lambda_{1}(y) d y .
$$

Due to (6), we see that for $x \in B$

$$
d\left(\hat{N}_{B} \nu_{1}\right)(x)=\lambda_{1}^{1-\frac{n}{p}}(x) \lambda_{2}^{\frac{n}{p}}(x) .
$$

Applying the first inequality from Corollary 6 with $\mu=\nu_{1}$, equality (7) and Hölder's inequality with the pair of conjugate exponents $p / n$ and $p /(p-n)$ 
we get

$$
\begin{aligned}
\int_{B} F(x)^{p} & \lambda_{1}(x) d x \\
& \leq \frac{p(p-1) \cdots(p-n+1)}{n !} \int_{B} f(x)^{n} F(x)^{p-n}\left(\hat{N}_{B}^{n-1} \nu_{1}\right)(d x) \\
& =\frac{p(p-1) \cdots(p-n+1)}{n !} \int_{B} f(x)^{n} \lambda_{2}(x)^{\frac{n}{p}} F(x)^{p-n} \lambda_{1}(x)^{1-\frac{n}{p}} d x \\
& \leq \frac{p(p-1) \cdots(p-n+1)}{n !}\left[\int_{B} f(x)^{p} \lambda_{2}(x) d x\right]^{\frac{n}{p}}\left[\int_{B} F(x)^{p} \lambda_{1}(x) d x\right]^{1-\frac{n}{p}},
\end{aligned}
$$

i. e.

$$
\left[\int_{B} F(x)^{p} \lambda_{1}(x) d x\right]^{\frac{n}{p}} \leq \frac{p(p-1) \cdots(p-n+1)}{n !}\left[\int_{B} f(x)^{p} \lambda_{2}(x) d x\right]^{\frac{n}{p}} .
$$

The densities

$$
\lambda_{1}(x)=x^{-k} \quad \text { and } \quad \lambda_{2}(x)=\frac{x^{p-k}}{(k-n)^{p / n}},
$$

with $k>n$, satisfy condition (6) and, in this case, the last inequality is equivalent with

$$
\left[\int_{B} x^{-k} F(x)^{p} d x\right]^{\frac{1}{p}} \leq K\left[\int_{B} x^{p-k} f(x)^{p} d x\right]^{\frac{1}{p}}
$$

where

$$
K=\sqrt[n]{\frac{p(p-1) \cdots(p-n+1)}{n !(k-n)}} .
$$

When $p=k>1$, the optimal constant in (8) is given by Hardy's inequality and equals $p /(p-1)$. This optimal constant is attained for $n=1$, while for $n>1$ the constant $K$ is strictly greater than the optimal.

Remark 8. When both $f$ and $-f$ satisfy the maximum principle, then the absolute value $|f|$ satisfies a similar condition, proven by Rao and Sikić (see $[6])$,

$$
|N f| \leq 2 M u+N\left[|f| \cdot \mathbf{1}_{\{|N f| \geq u\}}\right], \quad \text { for every } u \geq 0 .
$$


In that case, by using (9) instead of the maximum principle, one can prove the generalized potential inequality for absolute values: if $x \in X$ and $z>0$ are such that $z \leq|N f(x)| /(2 M)<+\infty$, then

$$
\begin{aligned}
g\left(\frac{1}{2 M}|N f(x)|\right)-g(z) \leq \frac{N(x, X)^{n-1}}{n !(2 M)^{n}}\left\{N\left[|f|^{n} g^{(n)}(|N f|) \mathbf{1}_{\bar{B}_{z}}\right](x)\right. \\
\left.-g^{(n)}(z) N\left[|f|^{n} \mathbf{1}_{\bar{B}_{z}}\right](x)\right\}+\sum_{i=1}^{n} \frac{1}{i !}\left(\frac{1}{2 M}|N f(x)|-z\right)^{i} g^{(i)}(z),
\end{aligned}
$$

where $\bar{B}_{z}=\{y \in X:|N f(y)| \geq z\}$.

Replacing the number $z$ with a function that satisfies $z(x) \leq|N f(x)| /(2 M)<+\infty$ for every $x \in \bar{B}=\{x \in X:|N f(x)|>0\}$ and integrating with respect to the variable $x$ one gets the integral version of the last inequality, for $C \subset \bar{B}, C \in \mathcal{B}(X)$,

$$
\begin{aligned}
& \int_{C}\left(g\left(\frac{1}{2 M}|N f(x)|\right)-g(z(x))\right) \mu(d x) \\
& \leq \frac{1}{n !(2 M)^{n}} \int_{C}\left(\int_{\bar{B}_{z(x)}}|f(y)|^{n} g^{(n)}(|N f(y)|) N(x, d y)\right) N(x, X)^{n-1} \mu(d x) \\
& -\frac{1}{n !(2 M)^{n}} \int_{C}\left(\int_{\bar{B}_{z(x)}}|f(y)|^{n} N(x, d y)\right) g^{(n)}(z(x)) N(x, X)^{n-1} \mu(d x) \\
& \quad+\sum_{i=1}^{n} \frac{1}{i !} \int_{C}\left(\frac{1}{2 M}|N f(x)|-z(x)\right)^{i} g^{(i)}(z(x)) \mu(d x) .
\end{aligned}
$$

In particular, for $C=\bar{B}_{z}$ and the constant function $z(x) \equiv z$ we get

$$
\begin{aligned}
& \int_{\bar{B}_{z}} g\left(\frac{1}{2 M}|N f(x)|\right) \mu(d x)-g(z) \mu\left(\bar{B}_{z}\right) \\
& \quad \leq \frac{1}{n !(2 M)^{n}} \int_{\bar{B}_{z}}|f(x)|^{n} g^{(n)}(|N f(x)|)\left(\hat{N}_{\bar{B}_{z}}^{n-1} \mu\right)(d x) \\
& -\frac{g^{(n)}(z)}{n !(2 M)^{n}} \int_{\bar{B}_{z}}|f(x)|^{n}\left(\hat{N}_{\bar{B}_{z}}^{n-1} \mu\right)(d x)+\sum_{i=1}^{n} \frac{g^{(i)}(z)}{i !} \int_{\bar{B}_{z}}\left(\frac{1}{2 M}|N f(x)|-z\right)^{i} \mu(d x) .
\end{aligned}
$$

\section{EXPONENTIAL CONVEXITY}

We will first construct linear functionals nonnegative on the set of $(n+1)$ convex functions by applying the generalized potential inequality, and its various forms, derived in the previous section. We then use the methods developed in [3] to generate new families of $n$-exponentially convex and 
exponentially convex functions by evaluating these functionals on families of functions satisfying similar properties.

Let us define linear functionals $A_{k}, k=1, \ldots, 6$, by

$$
\begin{aligned}
& A_{1}(g)=\frac{N(x, X)^{n-1}}{n ! M^{n}}\left\{N\left[\left(f^{+}\right)^{n} g^{(n)}(N f) \mathbf{1}_{B_{z}}\right](x)-g^{(n)}(z) N\left[\left(f^{+}\right)^{n} \mathbf{1}_{B_{z}}\right](x)\right\} \\
& +\sum_{i=1}^{n} \frac{1}{i !}\left(\frac{1}{M}(N f)(x)-z\right)^{i} g^{(i)}(z)-g\left(\frac{1}{M}(N f)(x)\right)+g(z), \\
& A_{2}(g)=\frac{1}{n ! M^{n}} \int_{B_{z}} f^{+}(x)^{n} g^{(n)}((N f)(x))\left(\hat{N}_{B_{z}}^{n-1} \mu\right)(d x) \\
& -\frac{g^{(n)}(z)}{n ! M^{n}} \int_{B_{z}}^{f_{z}} f^{+}(x)^{n}\left(\hat{N}_{B_{z}}^{n-1} \mu\right)(d x)+\sum_{i=1}^{n} \frac{g^{(i)}(z)}{i !} \int_{B_{z}}\left(\frac{1}{M}(N f)(x)-z\right)^{i} \mu(d x) \\
& \begin{array}{c}
-\int_{B_{z}} g\left(\frac{1}{M}(N f)(x)\right) \mu(d x)+g(z) \mu\left(B_{z}\right), \\
A_{3}(g)=\frac{N(x, X)^{n-1}}{n ! M^{n}}\left\{N\left[\left(f^{+}\right)^{n} g^{(n)}(N f) \mathbf{1}_{B}\right](x)-g^{(n)}(0+) N\left[\left(f^{+}\right)^{n} \mathbf{1}_{B}\right](x)\right\}
\end{array} \\
& +\sum_{i=0}^{n} \frac{1}{i ! M^{i}}(N f)(x)^{i} g^{(i)}(0+)-g\left(\frac{1}{M}(N f)(x)\right), \\
& A_{4}(g)=\frac{1}{n ! M^{n}} \int_{B} f^{+}(x)^{n} g^{(n)}((N f)(x))\left(\hat{N}_{B}^{n-1} \mu\right)(d x)-\int_{B} g\left(\frac{1}{M}(N f)(x)\right) \mu(d x) \\
& -\frac{g^{(n)}(0+)}{n ! M^{n}} \int_{B} f^{+}(x)^{n}\left(\hat{N}_{B}^{n-1} \mu\right)(d x)+\sum_{i=0}^{n} \frac{g^{(i)}(0+)}{i ! M^{i}} \int_{B}(N f)(x)^{i} \mu(d x), \\
& A_{5}(g)=\frac{N(x, X)^{n-1}}{n !(2 M)^{n}}\left\{N\left[|f|^{n} g^{(n)}(|N f|) \mathbf{1}_{\bar{B}_{z}}\right](x)-g^{(n)}(z) N\left[|f|^{n} \mathbf{1}_{\bar{B}_{z}}\right](x)\right\} \\
& +\sum_{i=1}^{n} \frac{1}{i !}\left(\frac{1}{2 M}|N f(x)|-z\right)^{i} g^{(i)}(z)-g\left(\frac{1}{2 M}|N f(x)|\right)+g(z), \\
& A_{6}(g)=\frac{1}{n !(2 M)^{n}} \int_{\bar{B}_{z}}|f(x)|^{n} g^{(n)}(|N f(x)|)\left(\hat{N}_{\bar{B}_{z}}^{n-1} \mu\right)(d x) \\
& -\frac{g^{(n)}(z)}{n !(2 M)^{n}} \int_{\bar{B}_{z}}|f(x)|^{n}\left(\hat{N}_{\bar{B}_{z}}^{n-1} \mu\right)(d x)+\sum_{i=1}^{n} \frac{g^{(i)}(z)}{i !} \int_{\bar{B}_{z}}\left(\frac{1}{2 M}|N f(x)|-z\right)^{i} \mu(d x) \\
& -\int_{\bar{B}_{z}} g\left(\frac{1}{2 M}|N f(x)|\right) \mu(d x)+g(z) \mu\left(\bar{B}_{z}\right) .
\end{aligned}
$$


The linear functionals $A_{k}$ depend on the choice of a function $f$, kernel $N$, measure $\mu$ and points $x$ and $z$, but we consider them given and omit from the notation. Also, we assume that a particular choice of $f, N, \mu, x$ and $z$ satisfies the assumptions of Theorem 2 (for $k=1$ ), Corollary 3 (for $k=2$ ), Corollary 5 (for $k=3$ or 4 ) and Remark 8 (for $k=5$ or 6 ).

We continue this section with few basic notions and results on exponential convexity that we will use.

Definition 2. A function $\psi: I \rightarrow \mathbb{R}$ is $n$-exponentially convex in the Jensen sense on $I$ if

$$
\sum_{i, j=1}^{n} \xi_{i} \xi_{j} \psi\left(\frac{x_{i}+x_{j}}{2}\right) \geq 0
$$

holds for all choices $\xi_{i} \in \mathbb{R}$ and $x_{i} \in I, i=1, \ldots, n$.

A function $\psi: I \rightarrow \mathbb{R}$ is $n$-exponentially convex if it is $n$-exponentially convex in the Jensen sense and continuous on $I$.

Definition 3. A function $\psi: I \rightarrow \mathbb{R}$ is exponentially convex in the Jensen sense on $I$ if it is $n$-exponentially convex in the Jensen sense for every $n \in \mathbb{N}$.

A function $\psi: I \rightarrow \mathbb{R}$ is exponentially convex if it is exponentially convex in the Jensen sense and continuous on $I$.

Definition of positive semi-definite matrices and some basic algebra gives us the following proposition

Proposition 9. If $\psi$ is an n-exponentially convex in the Jensen sense on $I$, then for every choice of $x_{i} \in I, i=1, \ldots, n$, the matrix $\left[\psi\left(\frac{x_{i}+x_{j}}{2}\right)\right]_{i, j=1}^{k}$ is a positive semi-definite matrix for all $k \in \mathbb{N}, k \leq n$. In particular, for all $k \in \mathbb{N}$, $\operatorname{det}\left[\psi\left(\frac{x_{i}+x_{j}}{2}\right)\right]_{i, j=1}^{k} \geq 0$ for all $k \leq n$.

Remark 10. It is known that $\psi: I \rightarrow \mathbb{R}$ is log-convex in the Jensen sense if and only if

$$
\alpha^{2} \psi(x)+2 \alpha \beta \psi\left(\frac{x+y}{2}\right)+\beta^{2} \psi(y) \geq 0
$$

holds for every $\alpha, \beta \in \mathbb{R}$ and $x, y \in I$. It follows that a function is log-convex in the Jensen sense if and only if it is 2-exponentially convex in the Jensen sense. Moreover, a function is log-convex if and only if it is 2-exponentially convex.

The next theorem will enable us to construct $n$-exponentially convex and exponentially convex functions. 
Theorem 11. Let $\Omega=\left\{g_{p}: p \in J\right\}$, where $J$ is an interval in $\mathbb{R}$, be a family of functions $g_{p}:(0,+\infty) \rightarrow \mathbb{R}$ such that the function $p \mapsto\left[z_{0}, \ldots, z_{n+1}\right] g_{p}$ is $m$-exponentially convex in the Jensen sense on $J$ for every $n+2$ mutually different points $z_{0}, \ldots, z_{n+1} \in(0,+\infty)$ and $A_{k}\left(g_{p}\right)$, for $1 \leq k \leq 6$, is well defined for every $p \in J$ (when $k=3$ or 4 , additional assumption is that $g_{p}^{(l)}(0+)$ is finite for every $l \leq n$ and $\left.p \in J\right)$. Then, the mapping $p \mapsto A_{k}\left(g_{p}\right)$ is an m-exponentially convex function in the Jensen sense on $J$. If the function $p \mapsto A_{k}\left(g_{p}\right)$ is continuous on $J$, then it is m-exponentially convex on $J$.

Proof. For $\xi_{i} \in \mathbb{R}$ and $p_{i} \in J, i=1, \ldots, m$, we define the function

$$
g(y)=\sum_{i, j=1}^{m} \xi_{i} \xi_{j} \frac{g_{\frac{p_{i}+p_{j}}{2}}}{2}(y) .
$$

Since $p \mapsto\left[z_{0}, \ldots, z_{n+1}\right] g_{p}$ is $m$-exponentially convex in the Jensen sense, we have

$$
\left[z_{0}, \ldots, z_{n+1}\right] g=\sum_{i, j=1}^{m} \xi_{i} \xi_{j}\left[z_{0}, \ldots, z_{n+1}\right] g_{p} \geq 0
$$

which, in turn, implies that $g$ is an $(n+1)$-convex function on $J$ (for $k=3$ and 4 it also holds that $g(0+)$ is finite). Therefore, by the generalized potential inequality,

$$
A_{k}(g)=\sum_{i, j=1}^{m} \xi_{i} \xi_{j} A_{k}\left(\frac{g_{\frac{p_{i}+p_{j}}{2}}}{2} \geq 0 .\right.
$$

We conclude that the function $p \mapsto A_{k}\left(g_{p}\right)$ is $m$-exponentially convex in the Jensen sense on $J$. If the function $p \mapsto A_{k}\left(g_{p}\right)$ is also continuous on $J$, then it is $m$-exponentially convex by definition.

Immediate consequence of the previous theorem and properties of exponentially convex functions is the following corollary.

Corollary 12. Let $\Omega=\left\{g_{p}: p \in J\right\}$, where $J$ is an interval in $\mathbb{R}$, be a family of functions $g_{p}:(0,+\infty) \rightarrow \mathbb{R}$ such that the function $p \mapsto$ $\left[z_{0}, \ldots, z_{n+1}\right] g_{p}$ is 2 -exponentially convex in the Jensen sense on $J$ for every $n+2$ mutually different points $z_{0}, \ldots, z_{n+1} \in(0,+\infty)$ and let the linear functionals $A_{k}, 1 \leq k \leq 6$, satisfy the same assumptions as in Theorem 11 . Then, the following statements hold:

(i) If the function $p \mapsto A_{k}\left(g_{p}\right)$ is continuous on $J$, then it is 2-exponentially convex and, thus, log-convex. 
(ii) If the function $p \mapsto A_{k}\left(g_{p}\right)$ is strictly positive and differentiable on $J$, then for every $p, q, r, s \in J$, such that $p \leq r$ and $q \leq s$, we have

$$
\mu_{p, q}^{k}(\Omega) \leq \mu_{r, s}^{k}(\Omega)
$$

where

$$
\mu_{p, q}^{k}(\Omega)= \begin{cases}\left(\frac{A_{k}\left(g_{p}\right)}{A_{k}\left(g_{q}\right)}\right)^{\frac{1}{p-q}}, & p \neq q \\ \exp \left(\frac{\frac{d}{d p} A_{k}\left(g_{p}\right)}{A_{k}\left(g_{p}\right)}\right), & p=q .\end{cases}
$$

for $g_{p}, g_{q} \in \Omega$.

Proof. (i) This is an immediate consequence of Theorem 11 and Remark 10.

(ii) By $(i)$, the function $p \mapsto A_{k}\left(g_{p}\right)$ is log-convex on $J$, that is, the function $p \mapsto \log A_{k}\left(g_{p}\right)$ is convex. Therefore

$$
\frac{\log A_{k}\left(g_{p}\right)-\log A_{k}\left(g_{q}\right)}{p-q} \leq \frac{\log A_{k}\left(g_{r}\right)-\log A_{k}\left(g_{s}\right)}{r-s}
$$

for $p \leq r, q \leq s, p \neq r, q \neq s$, which implies that

$$
\mu_{p, q}^{k}(\Omega) \leq \mu_{r, s}^{k}(\Omega), \quad k=1, \ldots, 6 .
$$

The cases $p=r$ and $q=s$ follow from (11) by taking limits $p \rightarrow r$ or $q \rightarrow s$.

Next, we present several families of functions that satisfy the assumptions of Theorem 11 and Corollary 12 and, in this way, we construct large families of exponentially convex functions.

Example 13. Consider a family of functions $\Omega_{1}=\left\{g_{p}: p \in \mathbb{R}\right\}$ given by

$$
g_{p}(y)= \begin{cases}\frac{e^{p y}}{p^{n+1}}, & p \neq 0 \\ \frac{y^{n+1}}{(n+1) !}, & p=0\end{cases}
$$

Similarly as in the proof of Theorem 11 , let us, for $\xi_{i} \in \mathbb{R}$ and $p_{i} \in \mathbb{R}$, $i=1, \ldots, m$, define the function

$$
g(y)=\sum_{i, j=1}^{m} \xi_{i} \xi_{j} g_{\frac{p_{i}+p_{j}}{2}}(y) .
$$

Since the function $p \mapsto \frac{d^{n+1}}{d y^{n+1}} g_{p}(y)=e^{p y}$ is exponentially convex (follows from the definition), we have that

$$
g^{(n+1)}(y)=\sum_{i, j=1}^{m} \xi_{i} \xi_{j} g_{\frac{p_{i}+p_{j}}{2}}^{(n+1)}(y)=\left(\sum_{i=1}^{m} \xi_{i} e^{p_{i} y / 2}\right)^{2} \geq 0
$$


is an $(n+1)$-exponentially convex function. Therefore

$$
0 \leq\left[z_{0}, \ldots, z_{n}\right] g=\sum_{i, j=1}^{m} \xi_{i} \xi_{j}\left[z_{0}, \ldots, z_{n}\right] g_{\frac{p_{i}+p_{j}}{2}},
$$

so $p \mapsto\left[z_{0}, \ldots, z_{n}\right] g_{p}$ is $m$-exponentially convex in the Jensen sense for every $m$. Using Theorem 11 we conclude that the mapping $p \mapsto A_{k}\left(g_{p}\right)$ is exponentially convex in the Jensen sense. It is easy to verify that this mapping is continuous (although the mapping $p \mapsto g_{p}$ is not continuous at $p=0)$, so it is exponentially convex.

For this family of functions, $\mu_{p, q}^{k}\left(\Omega_{1}\right)$ from (10) becomes

$$
\mu_{p, q}^{k}\left(\Omega_{1}\right)= \begin{cases}\left(\frac{A_{k}\left(g_{p}\right)}{A_{k}\left(g_{q}\right)}\right)^{\frac{1}{p-q}}, & p \neq q \\ \exp \left(\frac{A_{k}\left(i d \cdot g_{p}\right)}{A_{k}\left(g_{p}\right)}-\frac{n+1}{p}\right), & p=q \neq 0 \\ \exp \left(\frac{1}{n+2} \frac{A_{k}\left(i d \cdot g_{0}\right)}{A_{k}\left(g_{0}\right)}\right), & p=q=0,\end{cases}
$$

where $i d(y)=y$ is the identity function.

Example 14. Let $\Omega_{2}=\left\{g_{p}: p \in I_{k}\right\}$ be a family of functions defined by

$$
g_{p}(y)= \begin{cases}\frac{y^{p}}{p(p-1) \cdots(p-n)}, & p \notin\{0,1, \ldots, n\} \\ \frac{y^{j} \ln y}{(-1)^{n-j} j !(n-j) !}, & p=j \in\{0,1, \ldots, n\},\end{cases}
$$

where $I_{k}=(0,+\infty)$ for $k=1,2,5$ and 6 , and $I_{k}=(n,+\infty)$ for $k=3$ and 4. The mapping $p \rightarrow \frac{d^{n+1} g_{p}}{d y^{n+1}}(y)=e^{(p-n-1) \ln y}$ is exponentially convex (follows from the definition) and, arguing as in Example 13, we get that the mappings $p \mapsto A_{k}\left(g_{p}\right), 1 \leq k \leq 6$, are exponentially convex. In this case, the functions (10) are equal to

$$
\mu_{p, q}^{k}\left(\Omega_{2}\right)= \begin{cases}\left(\frac{A_{k}\left(g_{p}\right)}{A_{k}\left(g_{q}\right)}\right)^{\frac{1}{p-q}}, & p \neq q \\ \exp \left((-1)^{n} n ! \frac{A_{k}\left(g_{0} g_{p}\right)}{A_{k}\left(g_{p}\right)}+\sum_{k=0}^{n} \frac{1}{k-p}\right), & p=q \notin\{0,1, \ldots, n\}, \\ \exp \left((-1)^{n} n ! \frac{A_{k}\left(g_{0} g_{p}\right)}{2 A_{k}\left(g_{p}\right)}+\sum_{\substack{k=0 \\ k \neq p}}^{n} \frac{1}{k-p}\right), & p=q \in\{0,1, \ldots, n\} .\end{cases}
$$

Example 15. Let $\Omega_{3}=\left\{g_{p}: p \in(0,+\infty)\right\}$ be a family of functions given by

$$
g_{p}(y)= \begin{cases}\frac{p^{-y}}{(-\ln p)^{n+1}}, & p \neq 1 \\ \frac{y^{n+1}}{(n+1) !}, & p=1\end{cases}
$$


Since $p \mapsto \frac{d^{n+1} g_{p}}{d y^{n+1}}(y)=p^{-y}$ is the Laplace transform of a non-negative function (see [7]) it is exponentially convex. Arguing as in Example 13 we get that the mappings $p \mapsto A_{k}\left(g_{p}\right), 1 \leq k \leq 6$, are exponentially convex.

For this family of functions, $\mu_{p, q}^{k}\left(\Omega_{3}\right)$ from (10) becomes

$$
\mu_{p, q}^{k}\left(\Omega_{3}\right)= \begin{cases}\left(\frac{A_{k}\left(g_{p}\right)}{A_{k}\left(g_{q}\right)}\right)^{\frac{1}{p-q}}, & p \neq q \\ \exp \left(-\frac{A_{k}\left(i d \cdot g_{p}\right)}{p A_{k}\left(g_{p}\right)}-\frac{n+1}{p \ln p}\right), & p=q \neq 1, \\ \exp \left(-\frac{1}{n+2} \frac{A_{k}\left(i d \cdot g_{1}\right)}{A_{k}\left(g_{1}\right)}\right), & p=q=1 .\end{cases}
$$

Example 16. Let $\Omega_{4}=\left\{\Psi_{p}: p \in(0,+\infty)\right\}$ be a family of functions defined by

$$
g_{p}(y)=\frac{e^{-y \sqrt{p}}}{(-\sqrt{p})^{n+1}} .
$$

Since $p \mapsto \frac{d^{n+1} g_{p}}{d y^{n+1}}(y)=e^{-y \sqrt{p}}$ is the Laplace transform of a nonnegative function (see [7]), it is exponentially convex. Arguing as before, we get that $p \mapsto A_{k}\left(g_{p}\right), 1 \leq k \leq 6$, are exponentially convex functions.

For this family of functions, $\mu_{p, q}^{k}\left(\Omega_{4}\right)$ from (10) becomes

$$
\mu_{p, q}^{k}\left(\Omega_{4}\right)= \begin{cases}\left(\frac{A_{k}\left(g_{p}\right)}{A_{k}\left(g_{q}\right)}\right)^{\frac{1}{p-q}}, & p \neq q \\ \exp \left(-\frac{A_{k}\left(i d \cdot g_{p}\right)}{2 \sqrt{p} A_{k}\left(g_{p}\right)}-\frac{n+1}{2 p}\right), & p=q .\end{cases}
$$

\section{REFERENCES}

[1] N. Elezović, J. Pečarić, M. Praljak, Potential inequality revisited, I: General case, Math. Inequal. Appl. 15 (2012), 787-810.

[2] R. Ghulam, J. Pečarić, A. Vukelić, n-exponential convexity of divided differences and related Stolarsky type means, Math. Inequal. Appl. 16 (2013), 1043-1063.

[3] J. Jakšetić, J. Pečarić, Exponential convexity method, J. Convex Anal. 20 (2013), 181-197.

[4] J. Pečarić, J. Perić, Improvements of the Giaccardi and the Petrović inequality and related Stolarsky type means, An. Univ. Craiova Ser. Mat. Inform. 39 (2012), 65-75.

[5] J. Pečarić, F. Proschan, Y. L. Tong, Convex Functions, Partial Orderings and Statistical Application, Academic Press, San Diego, 1992.

[6] M. Rao, H. Šikić, Potential Inequality, Israel J. Math. 83 (1993), 97-127.

[7] J. L. Schiff, The Laplace transform. Theory and applications, Undergraduate Texts in Mathematics, Springer-Verlag, New York, 1999. 


\title{
ОБОПШТЕНО ПОТЕНЦИЈАЛНО НЕРАВЕНСТВО И ЕКСПОНЕНЦИЈАЛНА КОНВЕКСНОСТ
}

\author{
Невен Елезовиќ, Јосип Печариќ, Марјан Праљак \\ Р е $з$ и м е
}

Во овој труд го генерализираме потенцијалното неравенство кое е воведено во [6] и проширено на класата природно дефинирани конвексни функции во [1]. Генерализацијата е достигната со замена во доказот на потенцијалното неравенство, на Тејлоровата експанзија од прв ред на конвексна функција со Тајлоровата експанзија од ред $n$ на $(n+1)$ конвексна функција.

Освен тоа, користејќи методи развиени во [4] и [2] конструираме неколку фамилии од $n$-експоненцијални конвексни функции, со користење на линеарноста на обштеното потенцијално неравенство.

Faculty of Electrical Engineering and Computing, University of Zagreb, Unska 3, 10000 Zagreb, Croatia

E-mail address: neven.elez@fer.hr

Faculty of Textile Technology, University of Zagreb, Prilaz baruna FilIPOVIĆA 28A, 10000 ZAGREB, CROATIA

E-mail address: pecaric@element.hr

Faculty of Food Technology and Biotechnology, University of Zagreb, Pierottijeva 6, 10000 Zagreb, Croatia

E-mail address: mpraljak@pbf.hr 